\title{
TAXONOMIA E DISTRIBUIÇÃO ESTRATIGRÁFICA DOS OSTRACODES DA FORMAÇÃO QUIRICÓ, GRUPO AREADO (CRETÁCEO INFERIOR), BACIA SANFRANCISCANA, BRASIL
}

\author{
DERMEVALAPARECIDO DOCARMO, HENRIQUE ZIMMERMANNTOMASSI \\ \& SIMÃO BOLIVAR SAMPAIO GOMES DE OLIVEIRA \\ Instituto de Geociências, Universidade de Brasília, 70910-900, Brasília, DF, Brasil.derme@unb.br
}

\begin{abstract}
RESUMO - Este trabalho apresenta o primeiro estudo taxonômico detalhado dos ostracodes da bacia Sanfranciscana aliado a uma avaliação de sua paleoecologia e distribuição estratigráfica. As amostras analisadas são provenientes da Formação Quiricó, sete coletadas na Fazenda São Bento (Município de Carmo do Paranaíba) e quatro às margens da BR-040 (próximo ao rio do Sono), ambas as localidades no Estado de Minas Gerais. Foram identificadas quinze espécies de ostracodes não marinhos: Harbinia symmetrica? (Krömmelbein \& Weber, 1971), Harbinia sp. 1, Harbinia, sp. 2, Cypridea sp. 1, Bisulcocypridea sp. 1, Brasacypris ovum? Krömmelbein, 1965b, Brasacypris sp. 1, Ilyocypris sp. 1, Darwinula martinsi Silva , 1978, Darwinula sp. 1, Darwinula sp. 2, Darwinula sp. 3, Darwinula sp. 4, Wolburgiopsis plastica (Musacchio, 1970) e Wolburgiopsis chinamuertensis (Musacchio, 1970). Apenas Brasacypris sp. 1 e Wolburgiopsis chinamuertensis ocorrem em ambas as localidades. Darwinula martinsi, Brasacypris sp. 1 e Harbinia symmetrica? têm sido registradas em seqüências do Aptiano em outras bacias sedimentares do Brasil. Porém, as ocorrências de espécies exclusivas do Barremiano da Argentina, Wolburgiopsis plastica e Wolburgiopsis chinamuertensis indicam, pelo menos em parte, esta idade também para a deposição da porção da Formação Quiricó presentemente estudada. Desta forma, fica corroborada a idade Barremiano anteriormente admitida para a porção inferior desta formação.
\end{abstract}

Palavras-chave: Ostracode, bacia Sanfranciscana, Formação Quiricó, Cretáceo inferior.

ABSTRACT - TAXONOMY AND STRATIGRAPHIC DISTRIBUTION OF OSTRACODES FROM QUIRICÓ FORMATION, AREADO GROUP (LOWER CRETACEOUS), SANFRANCISCANA BASIN, BRAZIL. The taxonomic study of ostracodes from Sanfranciscana basin, their paleoecology and stratigraphic distribution is presented herein for the first time. Samples from the Quiricó Formation collected in two localities were analized: seven from São Bento Farm (Carmo do Paranaíba District) and four from a road cut at BR-040 (nearby Sono river), both localities in Minas Gerais State. Fifteen non-marine ostracod species were identified: Harbinia symmetrica? (Krömmelbein \& Weber, 1971), Harbinia sp. 1, Harbinia, sp. 2, Brasacypris ovum? Krömmelbein, 1965b, Brasacypris sp. 1, Cypridea sp. 1, Bisulcocypridea sp. 1, Ilyocypris sp. 1, Darwinula martinsi Silva , 1978, Darwinula sp. 1, Darwinula sp. 2, Darwinula sp. 3, Darwinula sp. 4, Wolburgiopsis plastica (Musacchio, 1970) and Wolburgiopsis chinamuertensis (Musacchio, 1970). Just Brasacypris sp. 1 and Wolburgiopsis chinamuertensis occur in both localities. D. martinsi, Brasacypris sp. 1 and $H$. symmetrica? have been recorded in Aptian sequences in other basins of Brazil. Although Wolburgiopsis plastica and Wolburgiopsis chinamuertensis, species restricted to Barremian strata of Argentine are indicating this age to the portion of the Formation Quiricó studied herein. So, the Barremian age admitted to the lower part of this formation is corroborated.

Key words: Ostracode, Sanfranciscana basin, Quiricó Formation, lower Cretaceous.

\section{INTRODUÇÃO}

Neste trabalho são apresentados os resultados de pesquisa focada na ocorrência de ostracodes em duas áreas da bacia Sanfranciscana onde afloram sedimentos da Formação Quiricó (Grupo Areado), respectivamente nos municípios de Carmo do Paranaíba e de João Pinheiro, Estado de Minas Gerais (MG).
Nestas duas localidades foram realizados perfis geológicos e coleta de amostras para estudo micropaleontológico com o objetivo de criar uma base para correlação bioestratigráfica na bacia Sanfranciscana. Os resultados aqui apresentados são inéditos e tratam da primeira publicação com ênfase na taxonomia e correlação bioestratigráfica baseada em espécies de ostracodes do Cretáceo da bacia Sanfranciscana. 


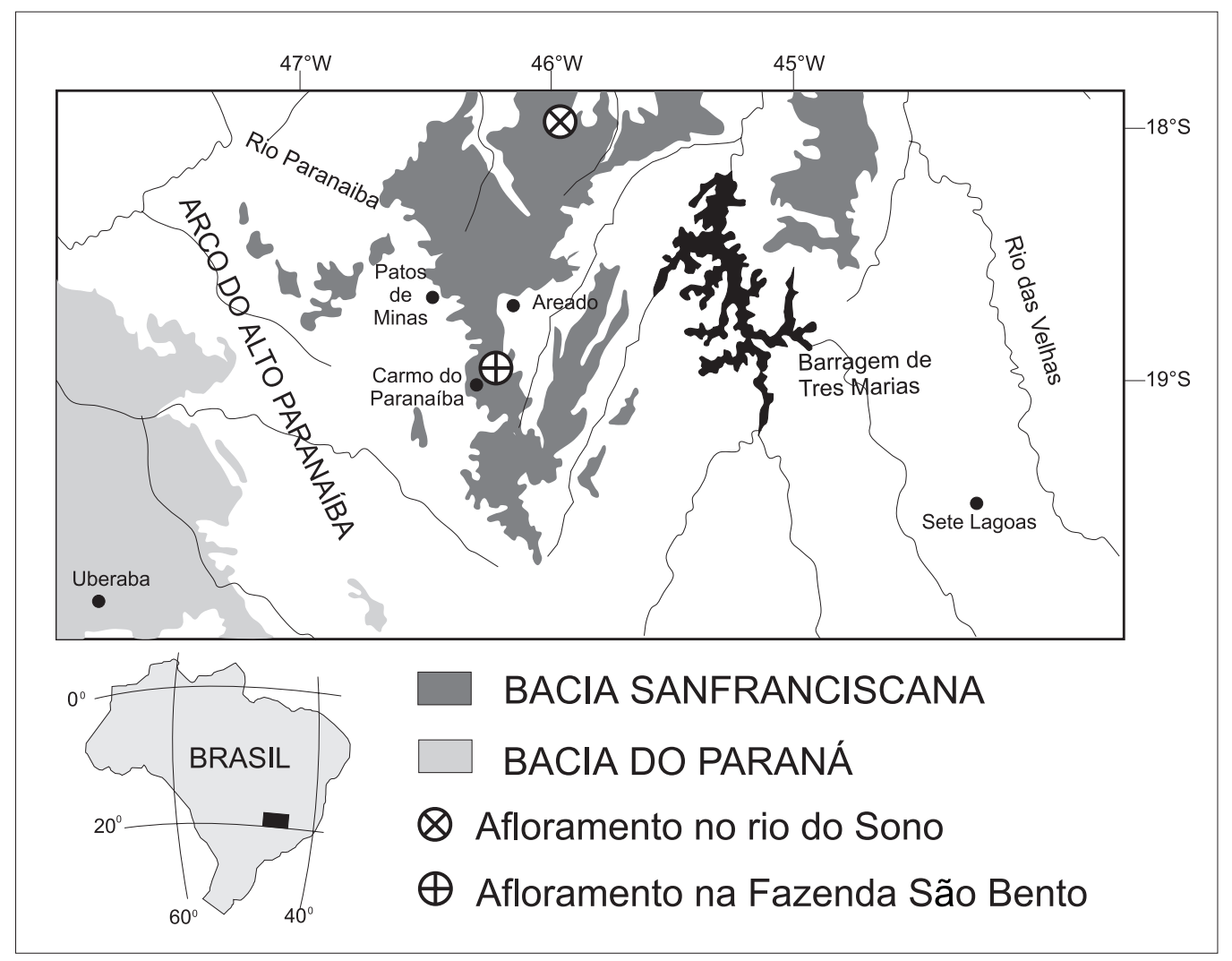

Figura 1. Mapa de localização da porção sul da bacia Sanfranciscana (adaptado de Seer et al., 1989 e Campos \& Dardenne, 1997b). Figure 1. Location map from the southern portion of Sanfranciscana basin (adapted from Seer et al., 1989 and Campos \& Dardenne, 1997b).

Com a continuidade dos estudos micropaleontológicos nesta bacia, afloramentos com melhor preservação devem proporcionar a recuperação de melhores exemplares e assim permitir o refinamento na descrição das espécies deixadas em nomenclatura aberta neste trabalho. Apesar deste aspecto preliminar da taxonomia, algumas considerações sobre o paleoambiente e a idade das seções estudadas são apresentadas.

\section{Bacia Sanfranciscana}

A bacia Sanfranciscana é subdividida em duas sub-bacias, Abaeté ao sul e, na porção centro-norte, Urucuia. Estas subbacias estão separadas pelo alto de Paracatu presente na região meridional da bacia Sanfranciscana (Campos \& Dardenne, 1997a).

Os afloramentos estudados localizam-se na região oeste do Estado de Minas Gerais (Figura 1). Segundo Campos \& Dardenne (1997a), a bacia Sanfranciscana nesta área compreende a sub-bacia de Abaeté. As seções do Cretáceo desta sub-bacia são constituídas por rochas dos grupos Areado, Mata da Corda e Urucuia (Figura 2). O Grupo Areado é atribuído ao Cretáceo Inferior e apresenta uma grande variação lateral de fácies, caracterizadas por depósitos de leque aluvial, fluvial entrelaçado, flúvio-deltaico, lacustre, campo de dunas e interdunas. Os grupos Mata da Corda e Urucuia são atribuídos ao Cretáceo Superior. O primeiro é caracterizado por rochas vulcânicas alcalinas, enquanto que outro é composto por arenitos com estratificação cruzada (Campos \& Dardenne, 1997b).
Os intervalos litoestratigráficos atribuídos ao Grupo Areado estão distribuídos por toda a extensão da bacia Sanfranciscana, sendo contínuos na sub-bacia Abaeté e descontínuos na sub-bacia Urucuia. Três formações compõem o Grupo Areado: Abaeté, Quiricó e Três Barras (Campos \& Dardenne, 1997b).

A Formação Quiricó, da qual provém o material estudado neste trabalho, é composta predominantemente por sedimentos pelíticos interpretados como resultantes de uma sedimentação lacustre, com folhelhos e siltitos esverdeados, rosados, ocres, violáceos ou avermelhados, que ocorrem interestratificados, apresentando rápidas variações de cores. Subordinadamente ocorrem intercalações de arenitos finos, médios e grossos, os quais são mais freqüentes na parte superior da seqüência. De maneira restrita ocorrem fácies de calcários micríticos cinza esverdeados dispostos em bancos maciços associados aos pelitos (Sgarbi, 1989 in Campos \& Dardenne, 1997b).

Apenas confinados à região de Varjão - São José de Geribá, no Município de Presidente Olegário, ocorrem folhelhos pretos ricos em matéria orgânica, refletindo um ambiente mais profundo. Esta fácies, além de apresentar uma distribuição restrita, mostra espessuras reduzidas (Kattah, 1991 in Campos \& Dardenne, 1997b).

De uma forma geral, pelitos predominam na base da unidade, enquanto as intercalações arenosas, a partir da porção mediana da seção, passam a ser comuns em direção ao topo. 
Além da proeminente estratificação plano-paralela, diversas estruturas sedimentares são observadas: laminações e estratificações cruzadas, marcas onduladas assimétricas, marcas de onda cavalgantes, moldes de pseudomorfos de sais, marcas de sola, concreções carbonáticas e canais de corte e preenchimento (Campos \& Dardenne, 1997b).

\section{Afloramentos estudados}

Os dois afloramentos da bacia Sanfranciscana são compostos por seções psamíticas com pelitos associados da Formação Quiricó (Figura 3). Toda a seqüência do afloramento próximo ao rio do Sono pertence à Formação Quiricó. Possui na base uma camada espessa e maciça de arenito branco, com estratificações cruzadas acanaladas e, em porção superior, estruturas de fluidização. A camada sotoposta é composta por níveis de siltito e argilito com estratificação planoparalela. Acima da camada pelítica, predomina um pacote de argilito esverdeado. Foram coletadas quatro amostras nesse afloramento (MP-204, MP-205, MP-206 e MP-207).

Na Fazenda São Bento, metassiltitos com seixos centimétricos do Grupo Bambuí (Pré-Cambriano) compõem o embasamento da sequiência do Cretáceo. Acima da unidade do Pré-Cambriano aflora a Formação Quiricó, constituída por camada de arenito grosso cinza-esverdeado na base. Acima deste nível há três camadas de argilito intercaladas com arenito. A camada inferior é a mais espessa e de cor rosada, com marcas onduladas e algumas lentes de arenito fino a médio, alguns carbonáticos. A camada média é de cor arroxeada e a superior é de cor rosada e também com marcas onduladas. A porção superior é marcada pelo arenito médio de cor avermelhada da Formação Três Barras, sendo recoberto pelo lamproito Mata da Corda. A amostragem é restrita ao intervalo da Formação Quiricó (amostras MP-109, MP-110, MP-111, MP-112, MP-113, MP-114 e MP-115).

\section{SISTEMÁTICAPALEONTOLÓGICA}

A sistemática supragenérica segue aquela apresentada por Whatley et al. (1993), exceto para as grafias das superfamilias Cypridoidea, Cytheroidea e de Darwinuloidea, que seguem recomendações da ICZN - International Commission on Zoological Nomenclature - (2000, Artigo 29.2). Vale salientar que este procedimento não é usual, porém trata-se de uma correção introduzida originalmente por Martin \& Davis (2001).

Os espécimes ilustrados neste trabalho encontram-se tombados na Coleção de Pesquisa do Laboratório de Micropaleontologia do Instituto de Geociências da Universidade de Brasília sob o prefixo "CP-". Para se referir à frequiência dos espécimes, adota-se os seguintes parâmetros para as ocorrências das espécies: rara, uma a cinco carapaças; comum, seis a dez carapaças; abundante, mais do que dez carapaças.

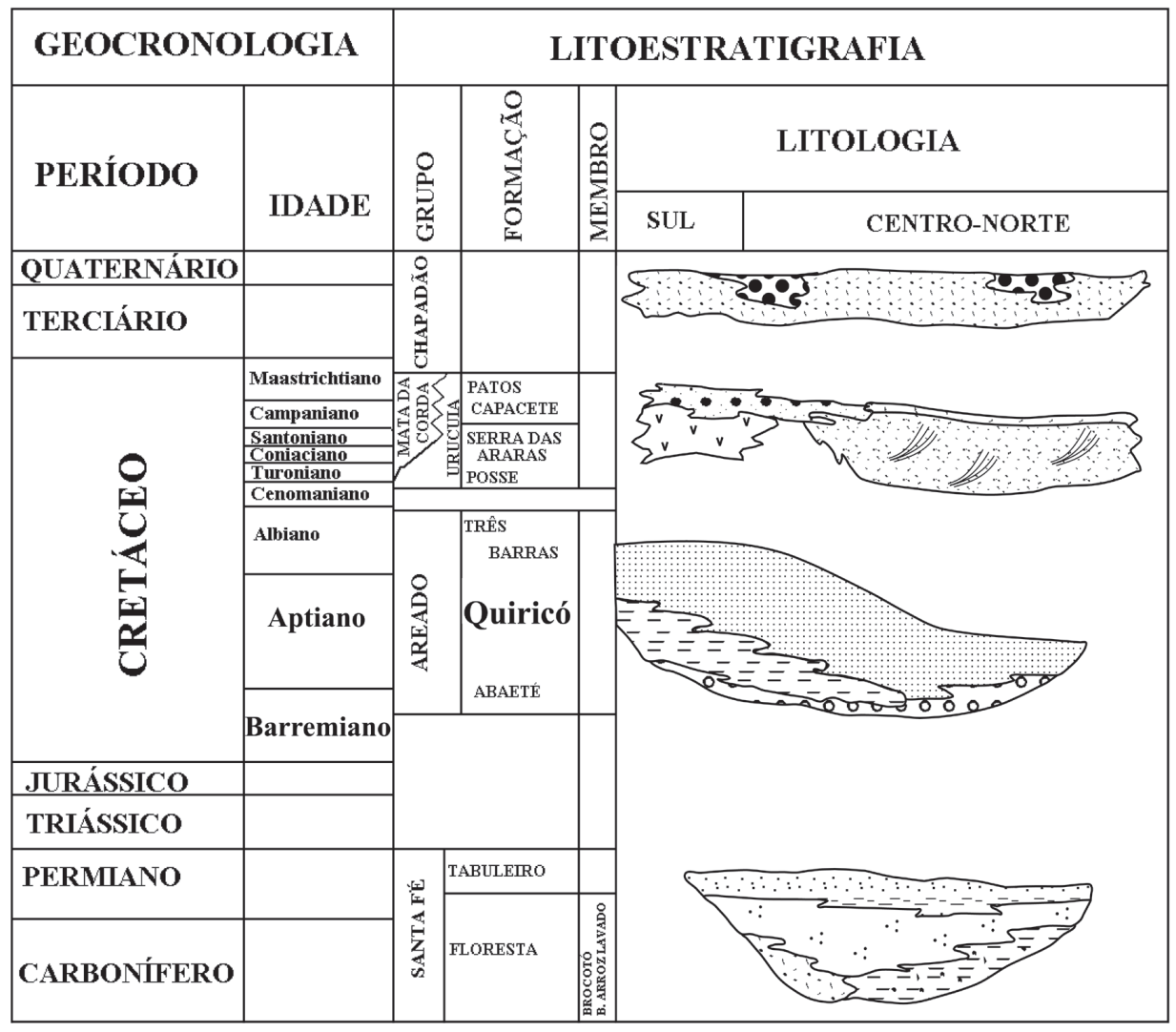

Figura 2. Coluna estratigráfica da bacia Sanfranciscana (adaptado de Campos \& Dardenne, 1997b).

Figure 2. Stratigraphic chart of Sanfranciscana basin (adapted from Campos \& Dardenne, 1997b). 
Na bacia Sanfranciscana pôde-se constatar a ocorrência de quinze espécies de ostracodes, das quais dez são indeterminadas: Harbinia symmetrica?, Harbinia sp. 1, Harbinia sp. 2, Brasacypris ovum?, Brasacypris sp. 1, Ilyocypris sp. 1, Cypridea sp. 1, Bisulcocypridea sp. 1, Darwinula martinsi, Darwinula sp. 1, Darwinula sp. 2, Darwinula sp. 3, Darwinula sp. 4, Wolburgiopsis plastica, Wolburgiopsis chinamuertensis. Além das espécies de ostracodes, foi registrada a ocorrência de alguns girogonites.

\author{
Classe OSTRACODA Latreille, 1806 \\ Ordem Podocopida Müller, 1894 \\ Subordem PODOCOPINA Sars, 1866 \\ Superfamília CYPRIDOIDEA Baird, 1845 \\ Família CYPRIDIDAE Baird, 1845 \\ Subfamília CYPRIDINAE Baird, 1845 \\ Gênero Harbinia Tsao, 1959 emend. Hou, 1984
}

1965a Hourcqia Krömmelbein, (non Hourcqia africana africana Krömmelbein, 1965a; H. africana confluens Krömmelbein \& Weber, 1971).

1972 Pattersoncypris Bate, p.380-381.

Espécie-tipo. Harbinia hapla Tsao, 1959.

Discussão. O gênero Harbinia foi atribuído originalmente à Família Cyprididae, porém sem designação de subfamília. O gênero Pattersoncypris, referido no presente trabalho como sinônimo junior de Harbinia, foi originalmente atribuído a Subfamília Cypridinae devido a excepcional preservação de apêndices em Harbinia micropapilosa (Bate, 1972), espécie fóssil do Cretáceo da bacia do Araripe, Brasil. Considerando a presente colocação do gênero Pattersoncypris em sinonímia de Harbinia, é possível atribuir este último à Subfamília Cypridinae. Vale salientar que Cypridea africana, originalmente designada como espécie-tipo do gênero Hourcqia, não é considerada um membro do gênero Harbinia Tsao, 1959 devido a presença de um bico característico do gênero Cypridea Bosquet, 1852.

Entretanto, as outras espécies atribuídas ao gênero Hourcqia por Krömmelbein \& Weber (1971) são aqui transferidas para Harbinia, uma vez que as mesmas são desprovidas de bico e compartilham várias características morfológicas com a espécie-tipo deste último gênero. Após a análise da espécie-tipo do gênero Pattersoncypris pode-se constatar qua a mesma apresenta similaridades marcantes com Harbinia, ou seja, formato, recobrimento, charneira adonte, presença de corcova e de impressões musculares típicas.

Harbinia symmetrica? (Krömmelbein \& Weber, 1971) (Figura 4.1)

1971 Hourcqia angulata symmetrica Krömmelbein \& Weber: p.36-37, est. 6, fig. 25.

Holótipo. BfB n.7797, material originalmente depositado no Serviço Geológico Alemão, porém atualmente o mesmo está abrigado pelo Museu Senckenberg, na cidade de Frankfurt am Main, Alemanha.
Localidade-tipo. Brasil, perfuração VGST-1-MA (Estado do Maranhão), amostra de calha aos 384 m, (Krömmelbein \& Weber, 1971).

Horizonte-tipo. Formação Codó, bacia do Maranhão, Aptiano (Krömmelbein \& Weber, 1971).

Homótipo. Carapaça juvenil (CP-21), comprimento de $682 \mu \mathrm{m}$, altura $394 \mu \mathrm{m}$ e largura $533 \mu \mathrm{m}$.

Localidade. Brasil, Município de Carmo do Paranaíba, afloramento na Fazenda São Bento (Figura 1).

Horizonte. Camada superior de argilito, amostra MP-111 (Figura 3b).

Ocorrência. Comum nas amostras MP-111 e MP-112 (Fazenda São Bento, Município de Carmo do Paranaíba); (Figura 3b).

Discussão. O estágio juvenil de Harbinia symmetrica? em questão guarda algumas semelhanças com o holótipo: morfologia subtriangular, maior altura na região antero-mediana e caimento simétrico da margem dorsal. Atribui-se o presente espécime a um estágio juvenil devido às suas menores dimensões quando comparado com o holótipo, o que resulta em pequenas diferenças morfológicas atribuíveis às diferenças ontogenéticas.

Distribuição geográfica e estratigráfica. Brasil, bacia do Maranhão, Formação Codó, Aptiano. Além da localidadetipo, esta espécie tem ocorrência registrada na perfuração PAF-2-MA, também no Estado do Maranhão (Krömmelbein $\&$ Weber, 1971). No presente trabalho, é registrada a primeira ocorrência na bacia Sanfranciscana, Formação Quiricó, Barremiano, Cretáceo Inferior.

Harbinia sp. 1

(Figura 4.2)

Hipótipo. Carapaça adulta CP-74, comprimento $1108 \mu \mathrm{m}$, largura $674 \mu \mathrm{m}$ e altura $532 \mu \mathrm{m}$.

Localidade. Brasil, Município de João Pinheiro, afloramento na BR-040, próximo ao rio do Sono (Figura 1).

Horizonte. Porção mediana da camada pelítica, amostra MP206 (Figura 3a).

Ocorrência. Abundante nas amostras MP-204, MP-205, MP206 e MP-207 (rio do Sono, Município de João Pinheiro) (Figura 3a).

Discussão. Harbinia sp.1 assemelha-se à Harbinia symmetrica, porém difere da mesma por apresentar maior altura na região central e extremidade anterior mais arredondada. A precariedade de preservação dificulta uma determinação mais precisa e por isso a espécie permanece em nomenclatura aberta.

Distribuição geográfica e estratigráfica. Brasil, bacia Sanfranciscana, Grupo Areado, Formação Quiricó, Barremiano, Cretáceo Inferior.

Harbinia sp. 2

(Figura 4.3)

? 1979 Hourcqia? GA G 14 Grosdidier: est. 12, figs. 66 a-c.

Hipótipo. Carapaça juvenil CP-20, comprimento $671 \mu \mathrm{m}$, altura $420 \mu \mathrm{m}$ e largura $379 \mu \mathrm{m}$. 
Localidade. Brasil, Município de Carmo do Paranaíba, afloramento na Fazenda São Bento (Figura 1).

Horizonte. Camada superior de argilito, amostra MP-114 (Figura $3 b$ ).

Ocorrência. Comum na amostra MP-114 (Fazenda São Bento, Município de Carmo do Paranaíba); (Figura 3b).

Discussão. O estágio juvenil de Harbinia sp. 2 presentemente ilustrado apresenta em linhas gerais morfologia semelhante à de Hourcqia? GA G 14 Grosdidier, 1979 (est. 12, figs 66 ac). Porém, difere da mesma por apresentar extremidade posterior mais afilada. O espécime ilustrado por Grosdidier (1979), distintamente maior $(1030 \mu \mathrm{m}$ de comprimento), pode tratarse de uma forma adulta e portanto permanece tentativamente na lista sinonímica.

Distribuição geográfica e estratigráfica. África, bacia do Gabão, Formação Mediéla, Aptiano Superior (Grosdidier, 1979). No presente trabalho registra-se pela primeira vez a ocorrência desta espécie na bacia Sanfranciscana, Grupo Areado, Formação Quiricó, Barremiano, Cretáceo Inferior.

\section{Gênero Brasacypris Krömmelbein, 1965b}

Espécie-tipo. Brasacypris ovum Krömmelbein, 1965b. Discussão. Originalmente, este gênero não teve família e subfamília determinada. Após uma detalhada comparação do gênero Brasacypris Krömmelbein, 1965b com Cyprinotus Brady, 1886, ainda que tentativamente, os mesmos foram atribuídos a mesma subfamília, ou seja, Cypridinae. Estudos futuros, se abrangerem o detalhamento das impressões musculares poderão chegar a uma conclusão sobre o assunto.

Brasacypris ovum? Krömmelbein, 1965b

(Figura 4.4)

? 1965 Brasacypris ovum Krömmelbein: p 198, est. 15, fig. 19.

Holótipo. SMF Xe 5369. Depositado no Museu Senckenberg, na cidade de Frankfurt am Main, Alemanha.

Localidade-tipo. Não especificada originalmente.

Horizonte-tipo. Bacia do Tucano, formações Itaparica e Candeias, amostra de superfície A-3.

Homótipo. Carapaça adulta CP-19, comprimento $1379 \mu \mathrm{m}$, altura $979 \mu \mathrm{m}$ e largura $507 \mu \mathrm{m}$.

Localidade. Brasil, Município de Carmo do Paranaíba, afloramento na Fazenda São Bento (Figura 1).

Horizonte. Camada superior de argilito, amostra MP-114 (Figura 3b).

Ocorrência. Raro na amostra MP-114 (Fazenda São Bento, Município de Carmo do Paranaíba); (Figura 3b).

Discussão. Os espécimes assemelham-se à Brasacypris ovum Krömmelbein 1965b, diferindo por apresentar um formato mais subtriangular do que ovóide e maior altura na região mediana. Porém, como as dimensões são similares e devido à precariedade da preservação, permanecem apenas tentativamente referidos como Brasacypris ovum?

Distribuição geográfica e estratigráfica. Brasil, bacia do

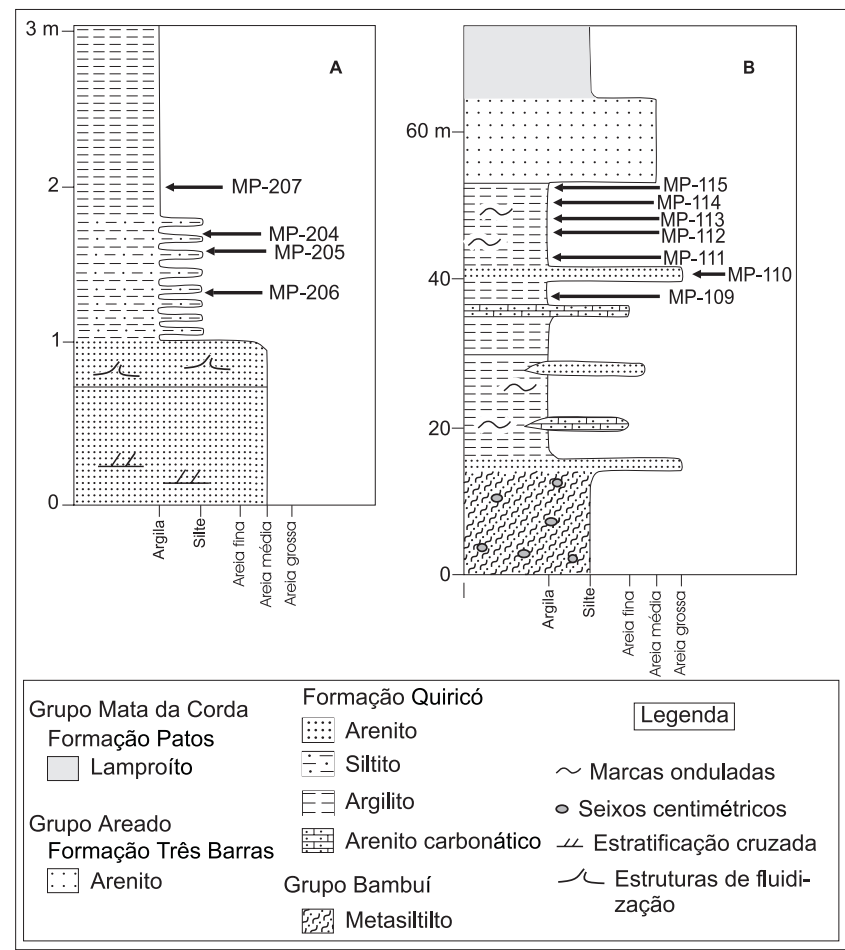

Figura 3. Afloramentos da bacia Sanfranciscana. A. Rio do Sono (Município de João Pinheiro, MG) e, B. Fazenda São Bento (Município de Carmo do Paranaíba, MG).

Figure 3. Outcrops in Sanfranciscana basin. A. Sono river (João Pinheiro District, MG) and, B. São Bento Farm (Carmo do Paranaíba District, MG).

Tucano, formações Itaparica e Candeias, Cretáceo Inferior (Krömmelbein, 1965b).

\section{Brasacypris sp. 1}

(Figura 4.5)

Hipótipo. Carapaça adulta CP-75, comprimento $1196 \mu \mathrm{m}$, altura $809 \mu \mathrm{m}$ e largura $649 \mu \mathrm{m}$.

Localidade. Brasil, Município de Carmo do Paranaíba, afloramento na Fazenda São Bento (Figura 1).

Horizonte. Porção mediana da camada pelítica, amostra MP206 (Figura 3a).

Ocorrências. Comum nas amostras MP-109, MP-111, MP-112, MP-114 e MP-115 (Fazenda São Bento, Município de Carmo do Paranaíba) e abundante nas amostras MP-204, MP-205, MP-206 e MP-207 (rio do Sono, Município de João Pinheiro). Discussão. Brasacypris sp. 1 foi comparada com Brasacypris ovum Krömmelbein, 1965b da qual difere pelo formato subretangular.

Distribuição geográfica e estratigráfica. Brasil, bacia Sanfranciscana, Grupo Areado, Formação Quiricó, Barremiano, Cretáceo Inferior.

Família ILYOCYPRIDIDAE Kaufmann, 1900 Subfamília ILYOCYPRIDINAE Kaufmann, 1900

Gênero Ilyocypris Brady \& Norman, 1889 Ilyocypris sp. 1

(Figura 4.6) 


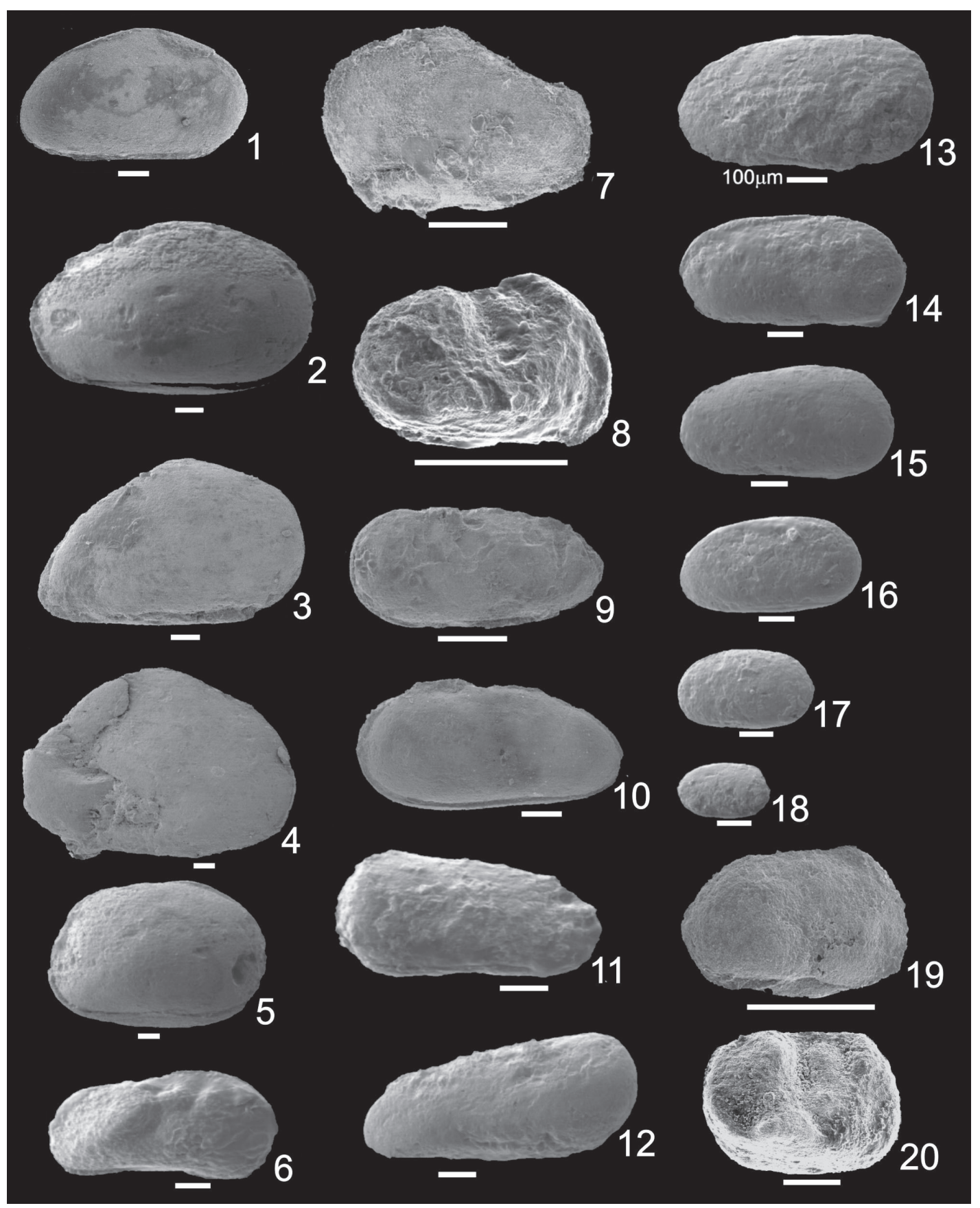

Figura 4. Ostracodes da bacia Sanfranciscana, Grupo Areado, Formação Quiricó. 1. Harbinia symmetrica?, vista lateral direita (CP-21). 2. Harbinia sp. 1, vista lateral direita (CP-74). 3. Harbinia sp. 2, vista lateral direita (CP-20). 4. Brasacypris ovum?, vista lateral esquerda (CP-19). 5. Brasacypris sp. 1, vista lateral direita (CP-75). 6. Ilyocypris sp. 1, vista lateral direita (CP-69). 7. Cypridea sp. 1, vista lateral esquerda (CP-23). 8. Bisulcocypridea? sp. 1, vista lateral direita (CP-10). 9. Darwinula martinsi, vista lateral direita (CP-15). 10. Darwinula sp. 1, vista lateral direita (CP-16). 11. Darwinula sp. 2, vista lateral direita (CP-71). 12. Darwinula sp.3, vista lateral esquerda (CP-70). 1318. Estágios ontogenéticos de Darwinula sp. 4. 13. Estágio adulto, vista lateral esquerda (CP-63). 14. Estágio A-1, vista lateral esquerda (CP-62). 15. Estágio A-2, vista lateral esquerda (CP-64). 16. Estágio A-3, vista lateral esquerda (CP-65). 17. Estágio A-4, vista lateral esquerda (CP-66). 18. Estágio A-5, vista lateral esquerda (CP-67). 19. Wolburgiopsis plastica, vista lateral direita (CP-24). 20. Wolburgiopsis chinamuertensis, vista lateral direita (CP-13). Escala gráfica $=100 \mu \mathrm{m}$.

Figure 4. Ostracodes from Sanfranciscana basin, Areado Group, Quiricó Formation. 1. Harbinia symmetrica?, lateral view, right valve (CP-21). 2. Harbinia sp. 1, lateral view, right valve (CP-74). 3. Harbinia sp. 2, lateral view, right valve (CP-20). 4. Brasacypris ovum?, lateral view, left valve (CP-19). 5. Brasacypris sp. 1, lateral view, right valve (CP-75). 6. llyocypris sp. 1, lateral view, right valve (CP-69). 7. Cypridea sp. 1, lateral view, left valve (CP-23). 8. Bisulcocypridea? sp. 1, lateral view, right valve (CP-10). 9. Darwinula martinsi, lateral view, right valve (CP-15). 10. Darwinula sp. 1, lateral view, right valve (CP-16). 11. Darwinula sp. 2, lateral view, right valve (CP-71). 12. Darwinula sp.3, lateral view, left valve (CP-70). 13-18. Ontogenetic stages of Darwinula sp. 4. 13. Adult stage, lateral view, left valve (CP63). 14. Stage A-1, lateral view, left valve (CP-62). 15. lateral view, left valve (CP-64). 16. Stage A-3, lateral view, left valve (CP-65). 17. Stage A-4, lateral view, left valve (CP-66). 18. Stage A-5, lateral view, left valve (CP-67). 19. Wolburgiopsis plastica, lateral view, right valve (CP-24). 20. Wolburgiopsis chinamuertensis, lateral view, right valve (CP-13). Scale bar $=100 \mu \mathrm{m}$. 
Hipótipo. Carapaça adulta CP-69, comprimento $661 \mu \mathrm{m}$, altura $320 \mu \mathrm{m}$ e largura $266 \mu \mathrm{m}$.

Localidade. Brasil, Município de João Pinheiro, afloramento na BR-040, próximo ao rio do Sono (Figura 1).

Horizonte. Porção inferior da camada de argilito, amostra MP207 (Figura 3a).

Ocorrências. Comum nas amostras, MP-204, MP-205, MP206 e MP-207 (rio do Sono, Município de João Pinheiro) (Figura 3a).

Discussão. Ilyocypris sp. 1 assemelha-se à Ilyocypris argentiniensis Musacchio \& Simeoni, 1991, da qual difere por apresentar a superfície da carapaça ornamentada com verrugas enquanto que I. argentiniensis apresenta ornamentação finamente reticulada. Também é comparável à I. riograndensis Musacchio \& Simeoni, 1991 do qual distingue-se por ser mais alongado e pela ornamentação com verrugas.

Distribuição estratigráfica. Brasil, bacia Sanfranciscana, Grupo Areado, Formação Quiricó, Barremiano, Cretáceo Inferior.

\section{Subfamília CYPRIDEINAE Martin, 1940 Gênero Cypridea Bosquet, 1852}

Espécie-tipo. Cypris granulosa Sowerby, 1836 (designada por Sylvester-Bradley, 1947).

Discussão. A atribuição deste gênero à Família Ilyocyprididae e à Subfamília Cyprideinae segue Moore \& Pitrat (1961).

\section{Cypridea sp. 1}

(Figura 4.7)

Hipótipo. Carapaça adulta CP-23, comprimento $536 \mu \mathrm{m}$, altura $308 \mu \mathrm{m}$ e largura $287 \mu \mathrm{m}$.

Localidade. Brasil, Município de Carmo do Paranaíba, afloramento na Fazenda São Bento (Figura 1).

Horizonte. Camada mediana de argilito, amostra MP-109 (Figura 3b).

Ocorrência. Comum na amostra MP-109 (Fazenda São Bento, Município de Carmo do Paranaíba); (Figura 3b).

Discussão. Apesar da má preservação, nota-se na margem antero-ventral a presença de uma projeção em forma de "bico", diagnóstica do gênero Cypridea.

Distribuição geográfica e estratigráfica. Brasil, bacia Sanfranciscana, Grupo Areado, Formação Quiricó, Barremiano, Cretáceo Inferior.

Gênero Bisulcocypridea (Sohn, 1969)

Espécie-tipo. Bisulcocypridea bicostata Sohn, 1969.

Discussão. Este gênero, originalmente, foi proposto na categoria de subgênero de Cypridea, porém, considerando o bem marcado formato bisulcado, em vista dorsal, pode-se distinguir o mesmo de Cypridea. Neste trabalho adota-se o grupo na categoria de gênero, procedimento já adotado por Colin \& Depeche (1997). Vale salientar que o aspecto bisulcado, justifica a inclusão deste gênero, na Família Ilyocyprididae, posição sem consenso entre os ostracodologistas.
Bisulcocypridea? sp. 1

(Figura 4.8)

Hipótipo. Carapaça juvenil CP-10, comprimento $349 \mu \mathrm{m}$, altura $210 \mu \mathrm{m}$ e largura $136 \mu \mathrm{m}$.

Localidade. Brasil, Município de Carmo do Paranaíba, afloramento na Fazenda São Bento (Figura 1).

Horizonte. Camada superior de argilito, amostra MP-115 (Figura 3b).

Ocorrência. Raro, apenas uma carapaça na amostra MP-115 (Figura 3b).

Discussão. Bisulcocypridea? sp. 1 é tentativamente atribuída a este gênero principalmente por apresentar dois sulcos na região ântero-mediana e um bico característico projetado a partir da margem ventral anterior. Uma descrição mais precisa para esta espécie ficou comprometida por tratar-se de estágio juvenil com má preservação e, por isso, a atribuição genérica é duvidosa.

Distribuição geográfica e estratigráfica. Brasil, bacia Sanfranciscana, Grupo Areado, Formação Quiricó, Barremiano, Cretáceo Inferior.

Superfamília DARWINULOIDEA Brady \& Norman, 1889 Família DARWINULIDAE Brady \& Norman, 1889

Gênero Darwinula Brady, \& Robertson, 1885 emend. Pinto \& Kotzian, 1961

Espécie-tipo. Polycheles stevensoni Brady \& Robertson, 1870. Discussão. Para este gênero, segue-se a sistemática adotada por Sohn (1987).

Darwinula martinsi Silva, 1978

(Figura 4.9)

1978 Darwinula martinsi Silva: p. 1028-30, est. 1, figs 1-2.

1990 Darwinula cf. martinsi Silva: Silva-Teles Jr. \& Viana, est. 2 , fig. 4.

1997 Darwinula martinsi Silva: Colin \& Depeche, est. 2 , fig. 18 .

2002 Darwinula martinsi Silva: Coimbra, Arai \& Carreño, est. 4 , fig. 32 .

Holótipo. O Holótipo desta espécie, originalmente depositado no setor de Paleontologia da Universidade Federal do Pernambuco, sob o número 42, foi perdido. Do Carmo et al. (no prelo) propõem os neótipos depositados junto ao Setor de Micropaleontologia do Museu de Paleontologia da Universidade Federal do Rio Grande do Sul, Porto Alegre, Estado do Rio Grande do Sul, números MP-O-1807 a MP-O1813.

Localidade-tipo. Brasil, Estado de Ceará, Município do Crato, Vila Lameiro, afloramento em afluente do rio Batateiras propriedade do Sr. Bessa Borges, $510 \mathrm{~m}$ de altitude (Silva, 1978). Horizonte-tipo. Bacia do Araripe, Formação Santana, Membro Crato, Aptiano. 
Homótipo. Carapaça juvenil CP-15, comprimento $719 \mu \mathrm{m}$, altura $302 \mu \mathrm{m}$ e largura $272 \mu \mathrm{m}$.

Localidade. Brasil, Estado de Minas Gerais, Município de Carmo do Paranaíba, afloramento na Fazenda São Bento (Figura 1).

Horizonte. Camada superior de argilito, amostra MP-114 (Figura 3b).

Ocorrência. Abundante na amostra MP-114 (Fazenda São Bento, Município de Carmo do Paranaíba); (Figura 3b).

Discussão. Darwinula martinsi apresenta um distinto aumento quase uniforme da altura em direção posterior, o tamanho e o recobrimento da valva esquerda em toda área de contato entre as valvas são características que permitem a determinação da espécie.

Distribuição geográfica e estratigráfica. Brasil, bacia do Araripe, Formação Santana, Membro Crato e base do Membro Ipubi (Silva, 1978, Silva-Telles Jr \& Viana, 1990, Colin \& Depeche, 1997) intervalo datado como Aptiano (Regali, 1990; Coimbra et al., 2002). No presente trabalho, bacia Sanfranciscana, Grupo Areado, Formação Quiricó, Barremiano, Cretáceo Inferior.

\section{Darwinula sp. 1}

(Figura 4.10)

1975 Darwinula sp. Musacchio \& Chebli: est. 11, figs. 1-2.

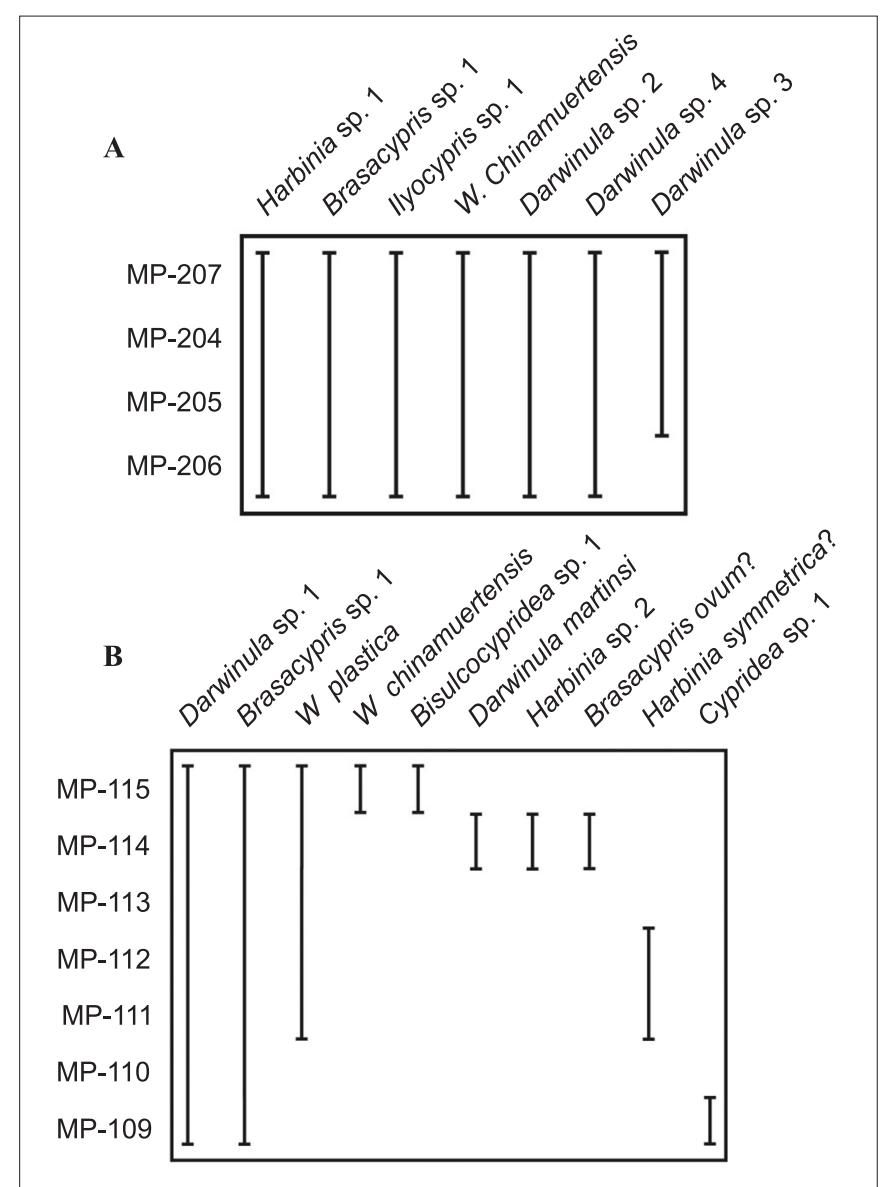

Figura 5. Distribuição estratigráfica e diversidade de espécies por afloramento: A, rio do Sono; B, Fazenda São Bento.

Figure 5. Stratigraphic distribution and diversity of species per outcrop: A, Sono river; B, São Bento Farm.
Hipótipo. Carapaça juvenil CP-16, comprimento $754 \mu \mathrm{m}$, altura $334 \mu \mathrm{m}$ e largura $271 \mu \mathrm{m}$.

Localidade. Brasil, Município de Carmo do Paranaíba, Fazenda São Bento (Figura 1).

Horizonte. Camada superior de argilito, amostra MP-114 (Figura $3 b)$.

Ocorrência. Abundante nas amostras MP-109, MP-110, MP111, MP-112, MP-113, MP-114 e MP-115 (Fazenda São Bento, Município de Carmo do Paranaíba) (Figura 3b).

Discussão. Darwinula sp. 1 foi apresenta grande similaridade Darwinula sp. Musacchio \& Chebli, 1975 (Grupo Chubut, Argentina), seja pelo formato subovalado alongado bem como pela distinta concavidade na margem ventral ântero-mediana.

Distribuição geográfica e estratigráfica. Argentina, Grupo Chubut, Formação Cerro Fortím, Membro Cerro Barcino, Cretáceo Inferior (Aptiano?) (Musacchio \& Chebli, 1975). Brasil, bacia Sanfranciscana, Grupo Areado, Formação Quiricó, Barremiano, Cretáceo Inferior.

\section{Darwinula sp. 2 \\ (Figura 4. 11)}

Hipótipo. Carapaça adulta CP-71, comprimento $586 \mu \mathrm{m}$, altura $304 \mu \mathrm{m}$ largura $234 \mu \mathrm{m}$.

Localidade. Brasil, Município de João Pinheiro, afloramento na BR-040, próximo ao rio do Sono (Figura 1).

Horizonte. Porção inferior da camada de argilito, amostra MP-207 (Figura 3a).

Ocorrência. Abundante nas amostras MP-204, MP-205, MP206 e MP-207 (rio do Sono, Município de João Pinheiro) (Figura 3a).

Discussão. Darwinula sp. 2 difere de Darwinula martinsi por apresentar a margem dorsal distinto caimento em direção a região anterior e maior altura em marcadamente mais próxima à extremidade posterior.

Distribuição estratigráfica. Brasil, bacia Sanfranciscana, Grupo Areado, Formação Quiricó, Barremiano, Cretáceo Inferior.

\section{Darwinula sp. 3 \\ (Figura 4.12)}

Hipótipo. Carapaça adulta CP-70, comprimento $785 \mu \mathrm{m}$, altura $296 \mu \mathrm{m}$ e largura $261 \mu \mathrm{m}$.

Localidade. Brasil, Município de João Pinheiro, afloramento na BR-40, próximo ao rio do Sono (Figura 1).

Horizonte. Porção inferior da camada de argilito, amostra MP-207 (Figura 3a).

Ocorrência. Abundante nas amostras MP-205 e MP-207 (rio do Sono, Município de João Pinheiro) (Figura 3a).

Discussão. Darwinula sp. 3 difere de Darwinula martinsi por apresentar a margem dorsal levemente convexa e com distinto caimento para a região anterior.

Distribuição geográfica e estratigráfica. Brasil, bacia Sanfranciscana, Grupo Areado, Formação Quiricó, Barremiano, Cretáceo Inferior. 
Darwinula sp. 4

(Figura 4.13-18)

Hipótipos. Série ontogenética completa, CP-62 a CP-67. Carapaça adulta CP-62, comprimento $648 \mu \mathrm{m}$, altura $261 \mu \mathrm{m}$ e largura $181 \mu \mathrm{m}$. CP-63, comprimento $618 \mu \mathrm{m}$, altura $273 \mu \mathrm{m}$ e largura $202 \mu \mathrm{m}$. CP-64, comprimento $584 \mu \mathrm{m}$. altura $259 \mu \mathrm{m}$ e largura $194 \mu \mathrm{m}$. CP-65, comprimento $507 \mu \mathrm{m}$, altura $236 \mu \mathrm{m}$ e largura $148 \mu \mathrm{m}$. CP-66, comprimento $412 \mu \mathrm{m}$, altura $203 \mu \mathrm{m}$ e largura $105 \mu \mathrm{m}$. CP-67, comprimento $274 \mu \mathrm{m}$, altura $157 \mu \mathrm{m}$ e largura $98 \mu \mathrm{m}$

Localidade. Brasil, Município de João Pinheiro, afloramento na BR-040, próximo ao rio do Sono (Figura 1).

Horizonte. Porção inferior da camada de argilito, amostra MP207 (Figura 3a).

Ocorrência. Abundante nas amostras MP-205, MP-206 e MP207 (rio do Sono, Município de João Pinheiro); (Figura 3a).

Discussão. Darwinula sp. 4 difere de Darwinula martinsi por apresentar as extremidades anterior e posterior arredondadas e a margem dorsal sub-retilínea com brusco arredondamento nas extremidades.

Distribuição geográfica e estratigráfica. Brasil, bacia Sanfranciscana, Grupo Areado, Formação Quiricó, Barremiano, Cretáceo Inferior.

\section{Superfamília CYTHEROIDEA Baird, 1850}

\section{Gênero Wolburgiopsis Uliana \& Musacchio, 1978}

Espécie-tipo. Wolburgia? neocretacea Bertels, 1972.

Discussão. A atribuição deste gênero em nível de família permanece incerta, porém, conforme apresentado originalmente, há semelhanças com a Família Limnocytheridae no que se refere às impressões musculares e na presença de dois sulcos subverticais. Apesar disso, a charneira antimerodonte constitui o elemento que deixa esta atribuição em nível de família questionável.

\section{Wolburgiopsis plastica (Musacchio, 1970)}

(Figura 4.19)

1970 Wolburgia plastica Musacchio: p. 302-306, est. 1, figs 1-5.

Holótipo. Depositado no Museu de La Plata (Argentina), número de tombamento MLP 11330; seção de paleozoologia de invertebrados (Musacchio, 1970).

Localidade-tipo. Argentina, Província de Neuquén, localidade denominada de cerro China Muerta (Musacchio, 1970).

Horizonte-tipo. Formação La Amarga, Membro "Margas y Calizas"; Barremiano, nível 35 (Musacchio, 1970).

Homótipo. Carapaça adulta de fêmea? CP-24, comprimento $475 \mu \mathrm{m}$, altura $294 \mu \mathrm{m}$ e largura $164 \mu \mathrm{m}$.

Localidade. Brasil, Município de Carmo do Paranaíba, afloramento na Fazenda São Bento (Figura 1).

Horizonte. Camada superior de argilito, amostra MP-111 (Figura 3b).
Ocorrência. Comum nas amostras MP-111, MP-114 e MP115 (Figura 3b).

Discussão. As dimensões diminutas, formato subtrapezoidal, a expansão ventral e os dois sulcos subverticais são características que permitem a determinação desta espécie.

Distribuição geográfica e estratigráfica. Argentina, Província Neuquén, Formação La Amarga, Barremiano (Musacchio, 1970). Brasil, bacia Sanfranciscana, Grupo Areado, Formação Quiricó, Barremiano, Cretáceo Inferior.

Wolburgiopsis chinamuertensis (Musacchio, 1970)

(Figura 4.20)

1970 Wolburgia chinamuertensis Musacchio: p. 306-308, est. 1, figs 6-8.

Holótipo. Depositado no "Museo de La Plata" (Argentina), número de tombamento MLP 11336, Seção de Paleozoologia de Invertebrados (Musacchio, 1970).

Localidade-tipo. Argentina, Província de Neuquén, cerro China Muerta (Musacchio, 1970).

Horizonte-tipo. Cerro China Muerta, Formação La Amarga, Membro "Margas y Calizas"; Barremiano, nível 38 (Musacchio, 1970).

Homótipo. Carapaça adulta CP-13, comprimento $340 \mu \mathrm{m}$, altura $217 \mu \mathrm{m}$ e largura $184 \mu \mathrm{m}$.

Localidade. Brasil, bacia Sanfranciscana, Grupo Areado, Formação Quiricó, Fazenda São Bento (Figura 3b).

Horizonte. Camada superior de argilito, amostra MP-115 (Figura 3b).

Ocorrência. Abundante nas amostras MP-204, MP-205, MP206 e MP-207 (rio do Sono, Município de João Pinheiro) e comum na amostra MP-115 (Fazenda São Bento) (Figs 3a-b).

Discussão. O tamanho diminuto, formato sub-retangular bissulcado e a ornamentação verrugosa permitem a determinação desta espécie.

Distribuição geográfica e estratigráfica. Argentina, Província de Neuquén, cerro China Muerta, Formação La Amarga, Membros "Margas y Calizas"; Barremiano, nível 38 (Musacchio, 1970). No presente trabalho, bacia Sanfranciscana, Grupo Areado, Formação Quiricó, Barremiano, Cretáceo Inferior.

\section{PALEOAMBIENTE E IDADE DA FORMAÇÃO QUIRICÓ}

Todas as espécies de ostracodes identificadas são nãomarinhas, portanto pode-se inferir o mesmo para o ambiente deposicional das seções da Formação Quiricó ora estudadas. Com relação aos ostracodes da bacia Sanfranciscana, o carbonato de cálcio das carapaças encontra-se muito dissolvido o que dificulta o trabalho de classificação. Porém as atribuições genéricas estão bem estabelecidas e, portanto as inferências paleoambientais também são precisas. A maior diversidade de espécies é notada no afloramento da Fazenda São Bento (Município de Carmo do Paranaíba, MG) (Figura 5). Ocorrências de microfósseis marinhos citadas por DiasBrito et al. (1999) para o Cretáceo da bacia Sanfranciscana, não foram registradas nas seções aqui estudadas. 
Conforme a coluna estratigráfica proposta por Campos \& Dardenne (1997b), a Formação Quiricó teria idade Barremiana/ Aptiana. A ocorrência de espécies tidas como do Aptiano, Harbinia symmetrica? (Aptiano da bacia do Maranhão, Brasil) e Darwinula martinsi (Aptiano da bacia do Araripe, Brasil), em estratos subjacentes àqueles onde se constata a ocorrências de espécies do Barremiano constitui um problema estratigráfico. Duas possibilidades parecem adequadas para explicar a situação, ou seja, as espécies $H$. symmetrica? e $D$. martinsi não são restritas ao Aptiano. A outra possibilidade seria o retrabalhamento das espécies do Barremiano, Wolburgiopsis plástica e Wolburgiopsis chinamuertensis (Barremiano da Argentina), porém não deve ser o caso, pois foram recuperados vários estágios ontogenéticos das mesmas, indicando assim, tratar-se de associação autóctone. Estes dados além de corroborarem em parte a idade Barremiana indicada por Campos \& Dardenne (1997b) para a porção inferior da Formação Quiricó, sugerem que ambos níveis estudados são restritos a esta porção da formação.

\section{AGRADECIMENTOS}

Agradecimentos aos professores José Elói Guimarães Campos e Marcel Auguste Dardenne por introduzir o primeiro autor ao estudo da bacia Sanfranciscana. Ao Conselho Nacional de Desenvolvimento Científico e Tecnológico-CNPq-pelo apoio financeiro concedido através da bolsa produtividade em pesquisa (processo 520309/99-5) e auxílio individual à pesquisa (processo 520053/00-1). Os demais autores agradecem ao CNPq pela concessão de suas bolsas de iniciação científica.

\section{REFERÊNCIAS}

Bate, R.H. 1972. Phosphatized ostracods with appendages from the Lower Cretaceous of Brazil. Paleontology, 15(3):379-393.

Bertels, A. 1972. Ostrácodos de água Dulce Miembro Inferior de la Formation Huantraico (Maastrichtiano inferior), Província de Neuquén, República Argentina. Ameghiniana 9(2):173-182.

Bosquet, J. 1852. Description des entomostracés fossiles des terrains tertiaires de la France e de la Belgique. Mémmoires Descr. Carte Géologie Neerland, 2:53-126.

Brady, G.S. 1886. Notes on Entomostraca collected by Mr. A. Haly in Ceilon. Journal of Linnean Society of Zoology, 19:239-317.

Brady, G.S. \& Robertson, D. 1870. The Ostracoda and Foraminifera of tidal rivers. With an analysis and descriptions of the British Ostracoda. Annals and Magazine of the Natural History, 4(31):1-33.

Campos, J.E.G. \& Dardenne, M.A. 1997a. Origem e evolução tectônica da bacia Sanfranciscana. Revista Brasileira de Geociências, 27(3):283-294.

Campos, J.E. G. \& Dardenne, M.A. 1997b. Estratigrafia e sedimentação da bacia Sanfranciscana: uma revisão. Revista Brasileira de Geociências, 27(3):269-282.

Coimbra, J.C.; Arai, M. \& Carreño, A.L. 2002. Biostratigraphy of the Lower Cretaceous microfossils from Araripe basin, northeastern Brazil. Geobios, 35:687-698.

Colin, J.-P. \& Depeche, F. 1997. Faunes d'ostracodes lacustres des bassins intra-cratoniques d'âge Albo-Aptian en Afrique de l'Ouest (Cameroun, Tchad) et au Brésil: Considérations d'ordre paléoécologiques et paléobiogéographiques. African Geoscience Review, 4(2/3):431-450.
Dias-Brito, D.; Pessagno Jr., E.A. \& Castro, J.C. 1999. Novas considerações cronoestratigráficas sobre o silexito a radiolários do sul da bacia Sanfranciscana, Brasil, e a ocorrência de foraminíferos plantônicos nesses depósitos: In: SIMPÓSIO SOBRE O CRETÁCEO DO BRASIL, 5, 1999. Boletim, Rio Claro, UNESP, p. 567-575.

Do Carmo, D.A.; Rafael, R.M.L.; Vilhena, R.M. \& Tomassi, H.Z. (no prelo). Redescrição de Theriosynoecum silvai e Darwinula martinsi, Membro Crato (Formação Santana), Cretáceo Inferior, bacia do Araripe, NE-Brasil. Revista Brasileira de Paleontologia.

Grosdidier, E. 1979. Principaux ostracodes marins de l'intervalle Aptien-Turonien du Gabon (Afrique Occidentale). Bulletin du Centre de Recherche et Exploration Elf-Aquitaine, 3(1):135 .

Hou, Y.T. 1984. Problems concerning the classification of the genera Harbinia, Sinocypris, Quadracypris and Nanxiongium (Ostracoda). Acta Micropaleontologica Sinica, 9:17-34.

International Commission on Zoological Nomenclature. 2000. International Code of Zoological Nomenclature. $4^{\mathrm{a}}$ ed. Londres, The International Trust for Zoological Nomenclature and The Natural History Museum, 306 p.

Krömmelbein, K. 1965a. Neue für Vergleiche mit West-Afrika wichtige ostracoden-Arten der Brasilianischen Bahia Serie (Ober-Jura? Unter-Kreide in Wealden-Fazies). Sonderdruck aus Sennckenbergiana Lethaea, 46:177-213.

Krömmelbein, K. 1965b. Ostracoden aus der nicht-marine UnterKreide ("Westafrikanischer Wealden") des Congo-Kusttenbeckens. Meyniana, 15:59-74.

Krömmelbein, K. \& Weber, R. 1971. Ostrakoden des "NordostBrasilianischen Wealden". Geologisches Jahrbuch, 115:1-93.

Martin, J.M. \& Davis, G.E., 2001. An updated classification of Recent Crustacea. Natural History Museum of Los Angeles County, Science Series, 39:1-124.

Moore, R.C. \& Pitrat, C.W. 1961. Treatise on Invertebrate Paleontology. Part Q, Arthropoda 3, Crustacea, Ostracoda. Lawrence, Geological Society of America/University of Kansas Press, $442 \mathrm{p}$.

Musacchio, E.A. 1970. Ostracodos de las superfamilias Cytheracea y Darwinulacea de la Formación La Amarga (Cretacico Inferior) en la Provincia de Neuquén, Republica Argentina. Revista de la Asociación Paleontológica Argentina, 7(4):301-317.

Musacchio, E.A. \& Chebli, G. 1975. Ostracodos marinos y carofitas del Cretacico Inferior en las provincias de Chubut y Neuquén, Argentina. Revista de la Asociación Paleontológica Argentina. 12(1):70-96.

Musacchio, E.A. \& Simeoni, M. 1991. Taxonomy of some Cretaceous non-marine ostracods of palaeobiogeographical interest. Neues Jahrbuch für Geologïe und Paläontologie Abhandlugen, 180(3):349-89.

Regali, M.S.P. 1990. Biocronoestratigrafia e paleoambiente do Eocretáceo das bacias do Araripe (CE) e Rio do Peixe (PB), NE - Brazil. In: SIMPÓSIO SOBRE A BACIA DO ARARIPE E BACIAS INTERIORES DO NORDESTE, 1, 1989. Atas, Crato, DNPM, p. 163- 172.

Sad, J.H.G.; Cardoso, R.N. \& Costa, M.T. 1971. Formações cretácicas em Minas Gerais. Revista Brasileira de Geociências, 1(1):2-13.

Seer, H.J.; Moraes, L.C. \& Fogaça, A.C.C. 1989. Roteiro geológico para a região de Formosa-Chumbo, Carmo do Paranaíba, MG. Boletim da Sociedade Brasileira de Geologia, Núcleo Minas Gerais, 9:57. 
Silva, D.M. 1978. Ostracodes da Formação Santana (Cretáceo Inferior - Grupo Araripe) Nordeste do Brasil - III - Nova espécie do gênero Darwinula Brady \& Robertson, 1885. In: CONGRESSO BRASILEIRO DE GEOLOGIA, 30, 1978. Anais, São Paulo, SBG, 2:1028-1031.

Silva-Telles Jr., A. \& Viana, M.S.S. 1990. Paleoecologia dos ostracodes da Formação Santana (bacia do Araripe): um estudo ontogenético de populações. In: SIMPÓSIO SOBRE A BACIA DO ARARIPE E BACIAS INTERIORES DO NORDESTE, 1, 1989. Atas, Crato, DNPM, p. 309-28.

Sohn, I.G. 1969. Nonmarine ostracodes of Early Cretaceous age from Pine Valley Quadrangle Nevada. Contributions to Paleontology, Geological Survey Professional Paper, 643-B:1-14.

Sohn, I.G. 1982. Dryelbidae n. fam. from continental Upper Jurassic and Lower Cretaceous rocks. In: R.H. Bate; E. Robinson \& L.M. Sheppard (eds.) Fossil and Recent Ostracodes. Chichester, Ellis Horwood, p. 305-325.
Sohn, I.G. 1987. The ubiquitous ostracode Darwinula stevensoni (Brady and Robertson, 1870), redescription of the species and lectotype designation. Micropaleontology, 33(2):150-163.

Sylvester-Bradley, P.C. 1947. The shell structure and evolution of the Mesozoic genus Cypridea. Quaterly Journal of the Geological Society, 103:7-9.

Tsao, L.P. 1959. Harbinia Tsao, 1959. In: M.A. Nechayeva et al. (eds.) Ostracods of lower chalk deposit of valley Sunlyao. Monographs of the Institute of Geology, Ministry of Geology, People's Republic of China, Series B (Stratigraphy and Palaeontology), 1(2):48-49.

Uliana, M.A. \& Musacchio, E.A. 1978. Microfossiles calcareous no-marinos del Cretacico Superior en Zampal, Provincia de Mendoza, Argentina. Ameghiniana, 15(1-2):111-135.

Whatley, R.C.; Siveter, D.J. \& Boomer, I.D. 1993. Arthropoda (Crustacea: Ostracoda). In: M.J. Benton (ed.) The Fossil Record. London, Chapman \& Hall, p. 343-356. 
